livraisons

d'Histoire

de l'Architecture

\section{Livraisons de l'histoire de l'architecture}

26 | 2013

Les ministres et les arts

\title{
Deux générations à côté du pouvoir :quelques remarques sur les arts chez les de Fourcy
}

Two generations on power's side: notes on the de Fourcys and the arts

Zwei Generationen in der Umgebung der Regierungsgewalt : einige

Bemerkungen zur Kunst der Familie de Fourcy

\section{Gabriele Quaranta}

\section{(2) OpenEdition}

Journals

Édition électronique

URL : http://journals.openedition.org/lha/339

DOI : $10.4000 /$ /ha.339

ISSN : 1960-5994

Éditeur

Association Livraisons d'histoire de l'architecture - LHA

Édition imprimée

Date de publication : 10 décembre 2013

Pagination : 105-122

ISSN : 1627-4970

\section{Référence électronique}

Gabriele Quaranta, « Deux générations à côté du pouvoir :quelques remarques sur les arts chez les de Fourcy ", Livraisons de l'histoire de l'architecture [En ligne], 26 | 2013, mis en ligne le 10 décembre 2015, consulté le 30 avril 2019. URL : http://journals.openedition.org/lha/339 ; DOI : 10.4000//ha.339 


\section{DEUX GÉNÉRATIONS À CÔTÉ DU POUVOIR : QUELQUES REMARQUES SUR LES ARTS CHEZ LES DE FOURCY*}

Lorsque le 24 mars 1594 Henri IV entrait enfin à Paris, plusieurs des chefs catholiques les plus influents avaient déjà rejoint le parti des Bourbons : ainsi, dans les rues de la capitale, un vieil homme de cour au sombre passé ligueur, monsieur de Humières, gouverneur de Picardie auparavant fidèle aux Guise, pouvait se retrouver à converser avec un personnage aux idées gallicaines, comme l'était l'intellectuel robin Jacques-Auguste de Thou. Ce dernier nous fait le récit de la rencontre et nous donne dans le même temps une anecdote savoureuse à propos du troisième personnage qui s'y trouvait en leur compagnie :

Monsieur d'Humières estoit fort genereux, $\&$ d'une fort ancienne $\&$ grande maison \& riche. Il me fit, le jour que le Roy prit Paris, le conte de Monsieur de Fourcy, car ayant trouvé Monsieur de Humières dans le jardin du baillage, \& ledit Fourcy avec luy, il me dit, connoissez-vous cet homme ; quand il vint a mon service, le plus malotru des mes valets de chien a un meilleur manteau qu'il n'avoit. Il a de l'Esprit. Le Roy Henri III. voyant la France perdüe, m'abandonna la Picardie, avec pouvoir de lever tout ce qu'il pouvoit lever. J'en donnay la charge à Fourcy, où il a fait ses affaires, et si bien, qu'il me parloit d'achetter une des mes terres six vingt mille francs. Il a gagné deux cens mille livres avec moy, \& moy je fuis engagé de deux cens mille escus. L'on ne sait d'où est Monsieur de Fourcy ${ }^{1}$.

Quelques jours plus tard, le 30 avril, ce Jean de Fourcy dont il est question ici, "trésorier de France en la généralité de Paris ", allait aussi obtenir l'office d'intendant des Bâtiments du roi $^{2}$. Son visage nous est connu par un très beau dessin

* Ces pages constituent un résumé d'un chapitre de ma thèse doctorale, L'Art du Roman. Peintures à sujet littéraire en France au XVII siècle, soutenue en juin 2013 en régime de cotutelle sous la direction de Mme C. Cieri Via (Sapienza Università di Roma) et Mme C. Nativel (Université Paris 1). Je profite pour remercier ici les éditeurs de la revue pour avoir accepté ma contribution, ainsi que Mlle Morgane Lecareux (Blois, Musée du Château), très chère amie et collègue, pour avoir révisé mon texte.

1. Thuana sive excerpta ex ore Jac. Aug. Thuani, per F.F.P.P., 1669, publiés dans Perroniana sive excerpta ex ore Cardinalis Perronii, per F.F.P.P., Genève, Apud Petrum Columnesium, M.DC.LXIX, p. 48.

2. Bernard Barbiche, "Henri IV et la surintendance des bâtiments ", Bulletin Monumental, $\mathrm{n}^{\circ}$ 142, 1984, p. 20. 


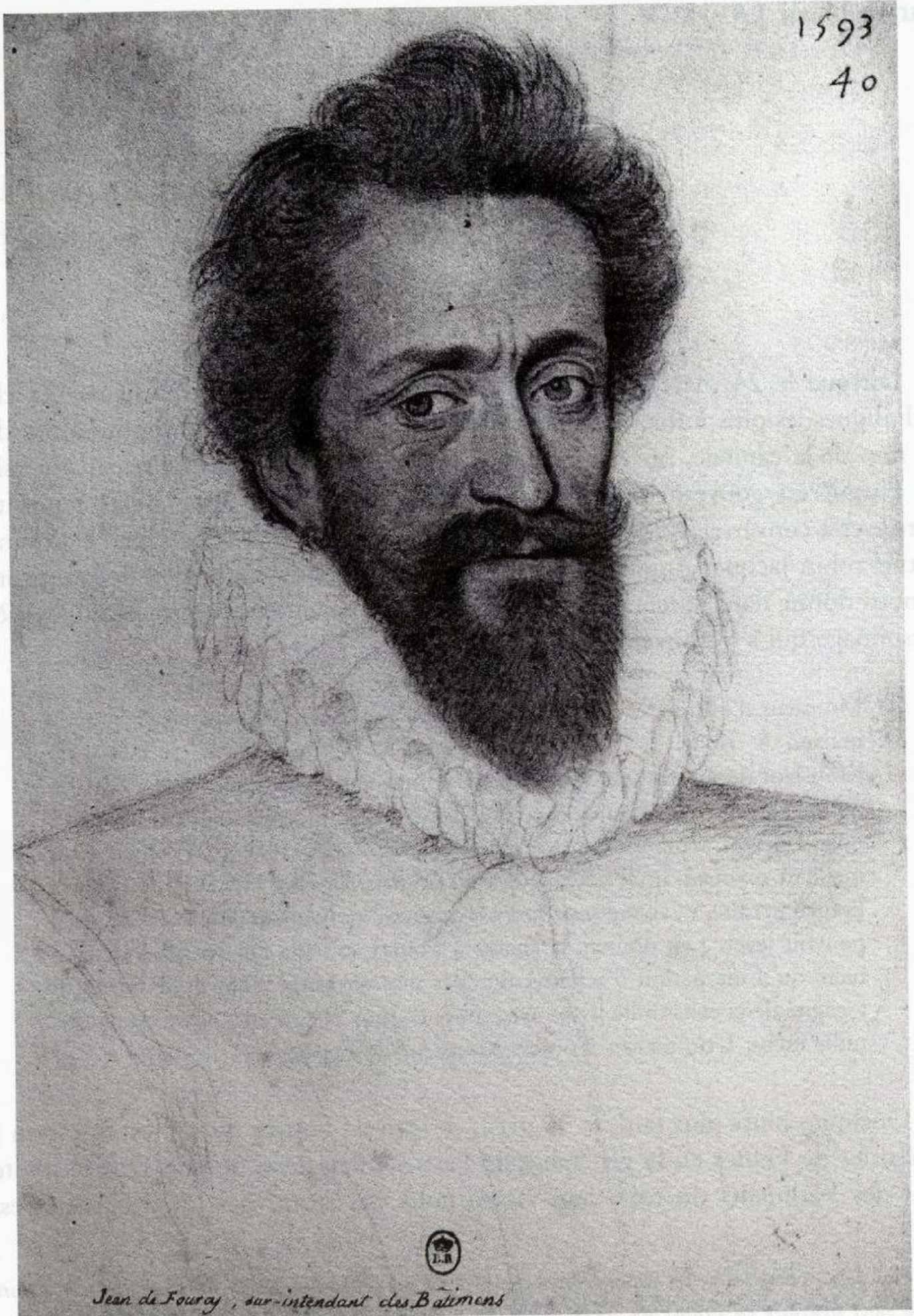

Ill. 1 : François Quesnel attr., Jean de Fourcy, surintendant des bastimens, Paris, BnF, Est. Res. NA-22 (5)BTE (fonds Gagnières).

(ill. 1) du célèbre fonds Gaignières : dans son catalogue, Étienne Moreau-Nélaton le donnait à François Quesnel, en y trouvant l'une de ses meilleures réussites ${ }^{3}$.

3. Étienne Moreau-Nélaton, Les Clouet et leurs émules, Paris 1924, t. II, fig. 238 et p. 38, t. III, p. 60. 
L'inscription qui l'accompagne nous le dit âgé de quarante-trois ans en 1593, mais comme le contrat de mariage de son père remonte à $1556^{4}$, à cette date il ne devait compter qu'entre trente-six et trente-sept ans. De plus il est nommé « surintendant ", un titre qu'il n'obtint qu'en 1621. De toute façon, le jeune ministre aux cheveux ébouriffés, aux beaux yeux clairs, au nez fort, au visage allongé, coincé par la fraise en dentelle, nous apparaît en homme à la figure pensive, voire mélancolique : son regard détourné, nous ne savons par quelle pensée, fermé dans une concentration chargée de doute. C'est l'image de quelqu'un qui a mûri suite aux soucis de sa charge, mais aussi d'un homme dont l'esprit sait ne pas se borner seulement à son travail quotidien d'administrateur.

\section{"Honori previa virtus » : Jean de Fourcy}

Son père, lui aussi nommé Jean, est dit dans une généalogie conservée à la Bibliothèque nationale de France " orfevre de Paris, escuyer et sieur de la Corbinière " 5 . Plus tard, il apparaît dans les sources comme conseiller du roi et commissaire des guerres $^{6}$ : c'était donc un bourgeois qui était arrivé à cumuler des charges, un fief, une petite fortune. Une situation sociale d'un niveau moyen, mais qui l'installe bien loin de l'indigence prétendue de son fils. D'ailleurs il s'était marié en février 1556 avec Marie Lecomte, fille d'un receveur général de Montpellier et trésorier de France en Languedoc dont le nom reste illisible sur le document généalogique ${ }^{7}$ : bien plus qu'un paysan ou petit bourgeois, qui sans doute n'aurait jamais souhaité une alliance avec un homme vraiment pauvre. Ainsi, le futur surintendant des Bâtiments ne venait pas de si bas, comme Jacques-Auguste de Thou voudrait nous le faire croire. Il avait sans doute cependant bien profité des guerres civiles pour faire fortune et pour mettre en place les fondements d'une carrière ensuite remarquable.

Si le récit de Jacques-Auguste de Thou est tourné si négativement, c'est bien pour remarquer l'origine très récente et "aventureuse " de cette fortune. L'image d'un Jean de Fourcy sans un sou, mais malin se dresse en évident contrepoint juste après celle de monsieur de Humières, qui était par contre " fort généreux, \& d'une fort ancienne \& grande maison \& riche ». À côté de ce dernier, Jean de Fourcy était l'homme nouveau, le roturier parvenu qui devait incarner cette classe de petits administrateurs qui jouait des coudes entre la noblesse d'épée, bouleversée par des décennies de guerre fratricide, et les robins érudits, provenant du Parlement et du barreau : une noblesse d'esprit, ces derniers, et de culture, rassemblée dans l'internationale République des Lettres ${ }^{8}$. Jacques-Auguste de Thou, qui était un représen-

4. Généalogie de la maison de Fourcy, Paris, BnF, Ms.Fr. 31039 (Cabinet d'Hozier).

5. Ibid.

6. De La Chesnaye Des Bois, Dictionnaire de la Noblesse, Paris, chez Duchesne, 1761, t. V, p. 151.

7. Ibid.; Généalogie de la maison de Fourcy, op. cit.

8. Marc Fumaroli, L'Âge de l'éloquence: rhétorique et res literaria de la Renaissance, Genève, Droz, 2008,890 p. 


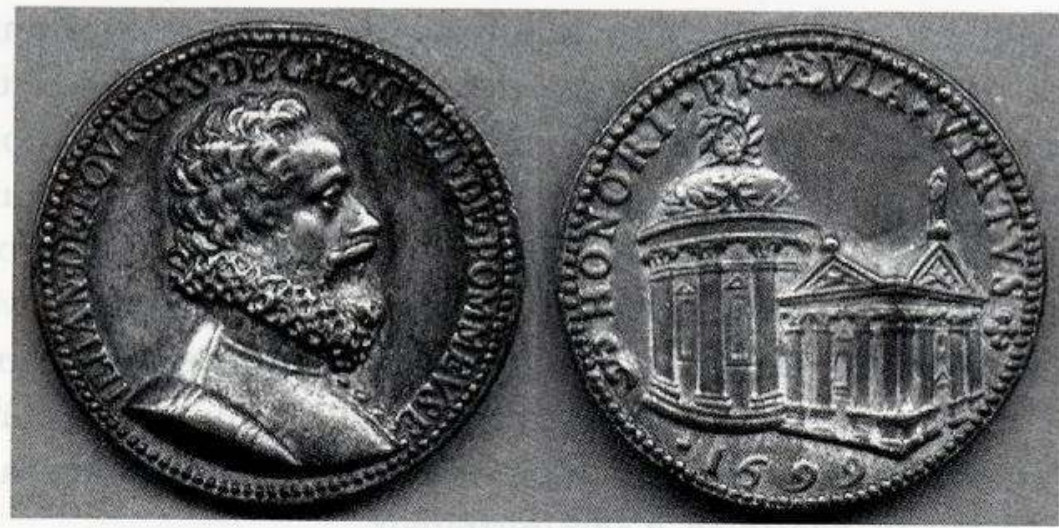

Ill. 2 : Médaille de Jean de Fourcy, 1599, argent, coll. part.

tant majeur de ce milieu, ne pouvait guère apprécier un personnage comme Jean de Fourcy.

D'ailleurs un écho explicite des bruits qui pouvaient courir autour du ministre est donné par un jeton frappé en 1599 (ill. 2). Un portrait est montré sur le recto : l'inscription "Iehan de Fourcy, s[ieur] de Chessy et de Pommeuse ", où ses fiefs sont nommés et non pas ses charges, est déjà intéressante car elle remarque les titres de noblesse plutôt que les fonctions d'administrateur. Mais c'est le verso qui est le plus éloquent car la devise " Honori prævia virtus " y accompagne la représentation de deux temples, l'un carré, le temple de la Vertu, précédant l'autre en plan central arrondi, le temple de l'Honneur, couronné d'une guirlande de laurier ${ }^{9}$. Cette même image fait aussi son apparition sur une médaille contemporaine d'Henri IV, seuls quelques détails en different ${ }^{10}$, et son concetto sera repris encore un siècle plus tard dans le portrait officiel de Louis XIV peint par Hyacinthe Rigaud en $1701^{11}$.

Jean de Fourcy n'avait pas choisi cette figuration par hasard. En affirmant que la vertu précède l'honneur - mieux : qu'elle en est la prémisse nécessaire - c'était bien son image d'homme vertueux qu'il voulait montrer : la vertu et rien d'autre l'avait mené tout au long de sa carrière et au rang dont il jouissait à la Cour. C'était une réponse directe aux médisances que n'importe quel petit aristocrate - de robe ou d'épée - pouvait sans doute adresser à un financier qui avait bien fait ses affaires et dont la carrière était en plein essor. Le lien entre le jeton de Jean de Fourcy et le récit de Jacques-Auguste de Thou est frappant, et nous allons retrouver cette dialectique entre les Fourcy et les milieux érudits de la robe parisienne bien des années plus tard.

9. Charles-Antoine Jombert, Catalogue raisonné de l'auvre de Sébastien Leclerc, Chevalier romain, Dessinateur \& Graveur du cabinet du Roi, Disposé par ordre historique et suivant l'année où chaque pièce a été gravée, depuis 1650 jusqu'en 1714 [...], première partie, Paris, Chez l'Auteur, 1774, p. 304. L'auteur se trompe ici avec un autre Jean de Fourcy, troisième du nom.

10. Jacques de Bie, La France Métallique, Paris, chez l'Auteur, 1636, pl. 89, n. XLII. Quelques détails different dans la façade du Temple de la Vertu.

11. Kirsten Ahrens, " "Honori praevia virtus". Une interprétation de l'architecture à l'arrière-plan du portrait officiel de Louis XIV peint par Rigaud en 1701 ", Gazette des Beaux-Arts, 6e série, 115, 1990 , p. 213-226. 
En 1599 la carrière de l'intendant Jean de Fourcy était encore à ses débuts. Nous ne nous y attarderons pas ici, car Bernard Barbiche l'a bien résumée dans son étude de 1984 en relevant et l'élargissement progressif des fonctions de la surintendance des Bâtiments - les manufactures des tapisseries, en 1599-1601, les grandes cérémonies royales - et l'augmentation en parallèle des revenus. L'ascension de Jean de Fourcy ne subit pas de contrecoups ni à la mort d'Henri IV, ni pendant les bouleversement des années 1617-1619. Alors que ses revenus atteignaient déjà le niveau d'un surintendant - 6000 livres -, lorsque Sully et son fils furent disgraciés en 1621 Jean se vit enfin confier cette même charge, jusqu'alors réservée à la haute noblesse et qu'il pourra transmettre, avant sa mort en 1625, à son fils Henri ${ }^{12}$. Au long des trois décennies qui le virent intendant, puis surintendant, Jean de Fourcy se trouva en spectateur privilégié et ensuite en acteur lui-même des choix artistiques de la Couronne ${ }^{13}$. Sa fréquentation des artistes était quotidienne : c'était Fourcy et le contrôleur Jean de Donon qui signaient tous les marchés pour les travaux - Sully apparait personnellement seulement à l'occasion des funérailles d'Henri IV et du couronnement de Marie de Médicis - et c'était lui qui prenait soin de ceux qui étaient engagés ${ }^{14}$. Les documents nous le montrent, par exemple, en charge du logement de Toussaint Dubreuil ${ }^{15}$ - qui travaillait à Saint-Germain-en-Laye et au Louvre - ou louant une dépendance de sa résidence parisienne à Charles Errard le père ${ }^{16}$.

Pourtant, face à une activité si intense, nous ne savons presque rien des commandes artistiques personnelles de Jean de Fourcy. Ses résidences ont disparu, la documentation nous en donne qu'une très pâle idée. Aucun marché pour des décorations n'est aujourd'hui connu. La chapelle familiale dans l'église Saint-GervaisSaint-Protais a été transformée au cours des siècles.

À Paris les Fourcy habitaient un hôtel particulier rue de Jouy, à mi-route entre leur paroisse et l'église jésuite Saint-Louis, à côté de l'hôtel d'Aumont ${ }^{17}$ : il a été remplacé par le lourd immeuble du Lycée Sophie Germain. Piganiol de la Force y fait allusion de façon fort synthétique en disant que «l'architecture en est un peu gothique, mais il a été réparé de façon que les dedans en sont bien pratiqués \& très-commodes $"{ }^{18}$. Pas question de tirer d'une telle remarque des informations à propos du style et de la chronologie du bâtiment, sauf que les héritiers de Jean n'avaient pas choisi de rebâtir à neuf leur résidence ou de l'adapter aux canons classicistes. Le Plan de Turgot (1739) montre sa structure comme tout à fait typique

12. Bernard Barbiche, "Henri IV et la surintendance des bâtiments ", op. cit., p. 19-20.

13. Voir par exemple l'épisode daté 1606 dans Maximilien de Béthune, duc de Sully, Mémoires du duc de Sully, Paris, Étienne Ledoux, 1827, t. IV, p. 522.

14. Le choix publié dans Marie-Antoinette Fleury, Documents du Minutier central de Paris - Peintres, sculpteurs et graveurs au XVII siècle (1600-1650), Monique Constans éd., Paris 2010, t. II, ad vocem Fourcy, Jean de, nous donne un large exemple de cette activité.

15. Ibid., n. 697.

16. Ibid., n. 768.

17. Alexandre Gady, Les Hôtels particuliers de Paris, Paris, Parigramme 2008, p. 186-189.

18. Jean-Aymar Piganiol de La Force, Description de Paris, Paris, T. Legras 1742, vol. IV, p. 297. 


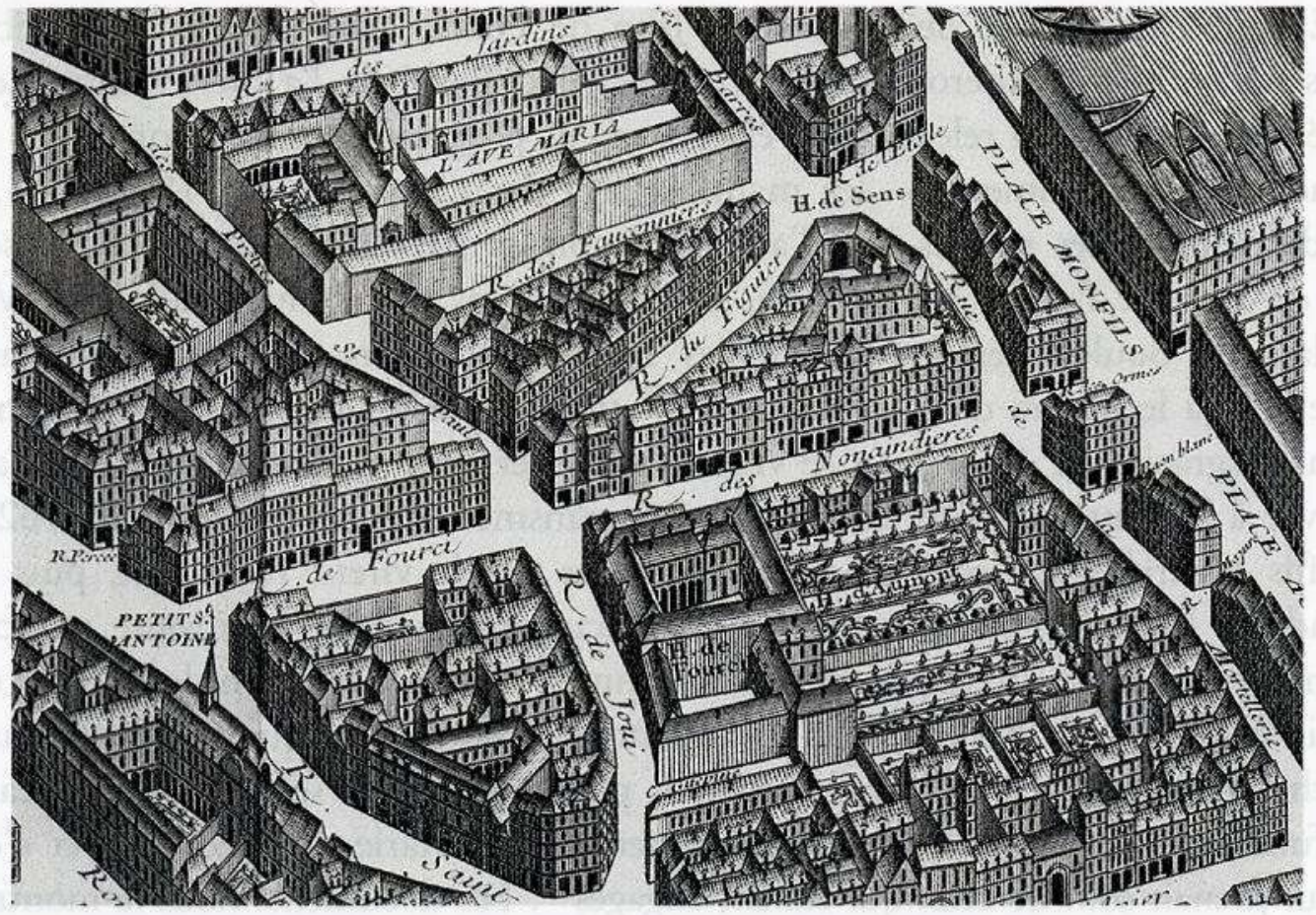

Ill. 3 : Louis Bretez, Claude Lucas, Plan de Paris, dit de Turgot, Paris 1739, planche 6, détail avec l'Hôtel de Fourcy.

(ill. 3) : un pavillon d'entrée, une cour carrée, un corps-de-logis qui s'ouvrait en deux petites ailes en retour sur le jardin, côtoyant celui de l'hôtel d'Aumont et divisé en deux longs parterres. Dressé sur deux étages, plus un niveau sous combles ouvert par des lucarnes, l'immeuble ne devait pas montrer une façade trop élaborée. Le Plan de Vaugondy, un peu plus tardif (1760), confirme le dessin général, mais montre un jardin qui semble avoir été entretemps réduit. La résidence des Fourcy pouvait donc bien montrer un caractère fin $\mathrm{XVI}^{\mathrm{e}}$ siècle - comme nous le voyons aujourd'hui à l'hôtel de Donon (Musée Cognacq-Jay), bâti après 1575 par le contrôleur général des Bâtiments du roi, Médéric de Donon - et remonter à une commission de Jean. Aucune grande décoration n'est signalée de façon claire dans son inventaire après décès, en 1626, sauf un cycle de huit tableaux encadrés dans la boiserie de la "Petite Salle", dont le sujet n'est pas noté ${ }^{19}$.

\section{Le château de Chessy}

Alors que la demeure parisienne des Fourcy nous reste encore méconnue, il sera possible d'en dire davantage à propos de leur résidence à la campagne, le château de Chessy, en Brie, resté dans la famille jusqu'au XVIII ${ }^{e}$ siècle (ill. 4). D'après Piganiol de La Force, qui le cite en 1718, suivi par les sources érudites et par la 


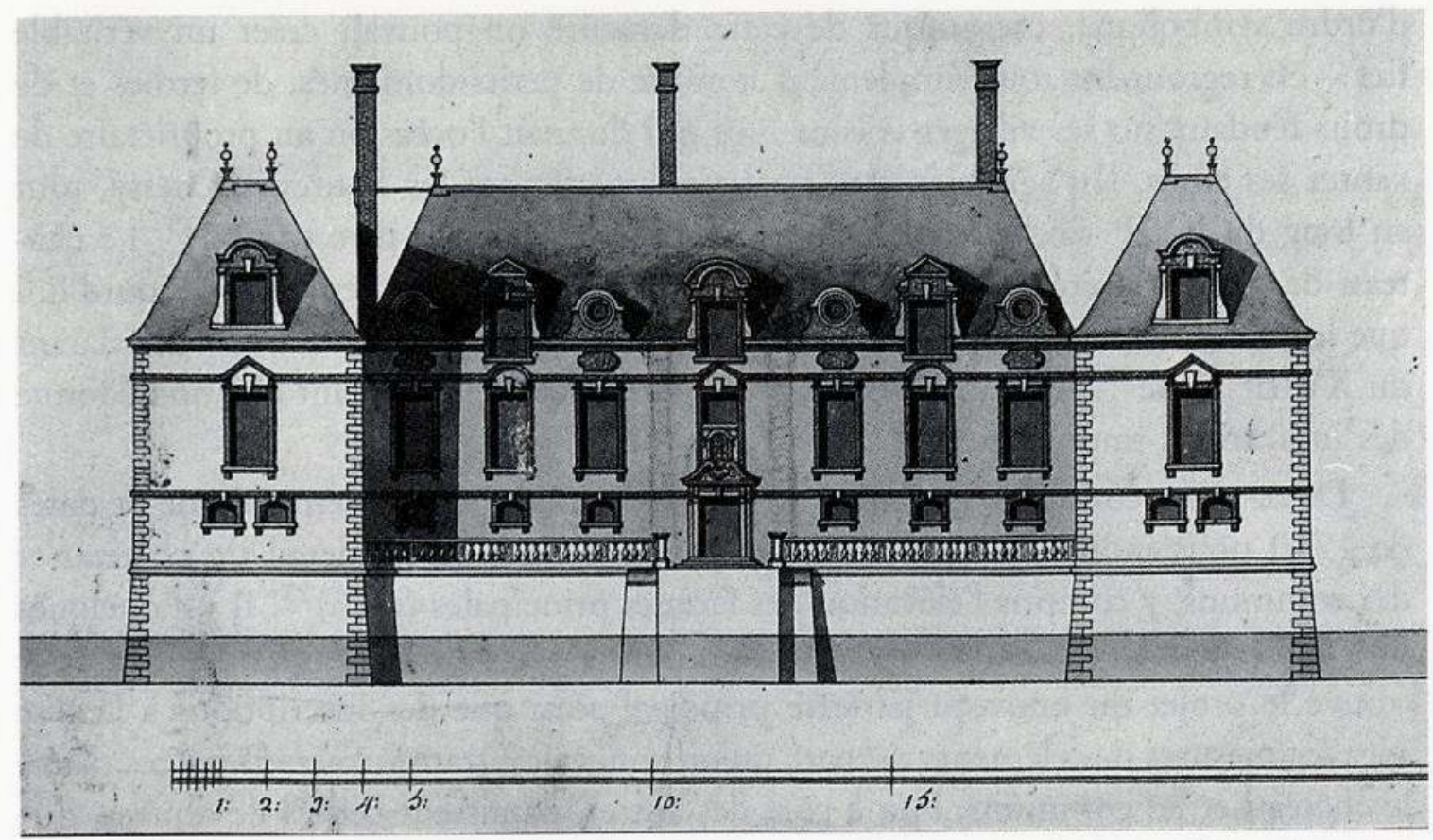

Ill. 4 : Anonyme, "Façade du Cotté de la Cour ", Plan du Château et Parc de Chessy, 1740, détail. Arch. dép. de Seine-et-Marne, Cartes et Plans, 14 FI 4759.

rare littérature critique, sa construction revenait à Jean ${ }^{20}$ : en effet, lorsqu'en 1611 on signe le contrat de mariage entre son fils Henri et Marie de La Grange-Trianon, le château existe déjà ${ }^{21}$. Un marché passé en décembre 1595 entre le ministre et Jean de Martin, habitant Coubron-en-Brie, portant sur une commande de 150000 briques au prix de 50 sols par millier ${ }^{22}$, pourrait bien se rattacher aux travaux de cette résidence : Coubron-en-Brie n'est qu'à une vingtaine de kilomètres de Chessy et dans ce document le commanditaire parait seul, alors que dans les marchés pour les commissions royales il agit normalement au nom du roi et il signe avec son aide Jean de Donon ${ }^{23}$.

Une résidence aux alentours de la capitale occupait sans doute une place importante parmi les intérêts du ministre, pour des raisons d'ordre économique aussi bien que social, car l'exploitation des ressources agricoles restait très importante et une résidence à la campagne était une démarche obligée pour n’importe quelle famille aristocratique, notamment pour les financiers, alors que la noblesse d'épée restait encore très liée aux châteaux ancestraux ${ }^{24}$. Mais il y avait aussi une raison

20. Jean-Aymar Piganiol de la Force, Nouvelle description de la France, Paris, F. Delaulne, 1718, vol. II, p. 499-500 ; Thierry Lhuillier, "Chessy ", Almanach historique, topographique et statistique du département de Seine-et-Marne et diocèse de Meaux, n 24, 1884, p. 22-130; Sylvain Kerspern, La peinture du XVII siècle en Brie, thèse de doctorat sous la dir. de Daniel Ternois, Université Paris 1-Panthéon Sorbonne, 1990, p. 264-266.

21. Chessy, Paris, BnF, Ms. Fr. 26301 (Collection Joult).

22. Arch. nat., MC, ET XIX 332, 6 décembre 1595, Marché entre Jean de Fourcy et Jean de Martin.

23. Documents du Minutier Central, op. cit., n. 408-4009, 411, 829, 833, 993, 1000-1001, etc.

24. Jean-Marie Pérouse de Montclos, Histoire de l'architecture française. De la Renaissance à la Révolution, Paris, Mengès 1989, p. 215-247. 
d'ordre symbolique, car autour de cette demeure on pouvait créer un véritable fief - en regroupant tout simplement nombre de petits domaines, de fermes et de droits féodaux sur les villages voisins - ce qui donnait l'occasion au propriétaire de vanter ses titres. Il s'agit bien de l'opération menée par les Fourcy à Chessy, tout au long du XVII ${ }^{\mathrm{e}}$ siècle, jusqu'à l'obtention en 1672 d'un titre comtal ${ }^{25}$. Le château de Chessy a été détruit peu après la Révolution et il n'en reste aujourd'hui que le colombier, un petit pavillon faisant partie des communs et la glacière datant du XVIII ${ }^{\text {e }}$ siècle $^{26}$. La documentation est plutôt tardive, pourtant elle nous donne des indications importantes.

Deux plans conservés aux archives départementales de Seine-et-Marne et datés de 1740 nous montrent l'ensemble du domaine ainsi qu'un détail du château et des communs, y compris l'élévation des façades principales (ill. 5) ${ }^{27}$. Il y a quelques différences entre eux, mais tous deux montrent l'usage pratique qu'on y fit : on y trouve le projet du nouveau parterre principal ainsi que des inscriptions à l'encre avec les mesures des éléments du parc qu'on songeait à transformer. Dans ces plans, le château et les communs, l'un à côté des autres, paraissent cernés de douves, formant deux îlots carrés, accessibles par des ponts. Un grand canal se développait à gauche, alors qu'un axe principal, organisé en parterres, traversait le parc et la demeure de l'entrée jusqu'au village. Un deuxième axe bordait le premier, du canal jusqu'à l'orangerie en abritant en son milieu la statue de Gilles Guérin, Louis XIV terrassant la Fronde, aujourd'hui à Chantilly ${ }^{28}$. La littérature érudite atteste des travaux d'agrandissement menés par Henri II de Fourcy au milieu du XVII ${ }^{e}$ siècle et l'organisation du parc pourrait bien remonter à cette époque, mais la structure du château peut nous ramener au temps de Jean : par son plan en double île au milieu d'un véritable bassin, la résidence seigneuriale semble vaste et imposante, mais ses dimensions réelles étaient en effet modestes et ses façades montrent des traits archaïques.

Au-dessus de sa plateforme, le château se dessinait sur un plan carré, avec un corps de logis principal, flanqué des deux pavillons : de-là deux ailes en retour sur cour se développaient, elles aussi gardées par deux pavillons du côté de l'entrée, qui restait libre. Cette structure suivait un schéma précis : deux carrés inscrits, non concentriques mais coïncidant sur l'axe d'entrée. Le plus grand - 25 toises $(48,725 \mathrm{~m})$ - dessinait le périmètre extérieur, y compris la saillie des pavillons, alors que le plus petit - 15 toises $(29,235 \mathrm{~m})$ - déterminait l'ampleur de la cour d'honneur. Le dessin des façades (ill. 4) montre que le château s'élevait sur trois

25. De La Chesnaye Des Bois, Dictionnaire de la Noblesse, op. cit., t. V, p. 152.

26. Arch. dép. Seine-et-Marne, Cartes et Plans, 4P37/1350, Cadastre Napoléonien, 1824-1830, Chessy : ce plan cadastral montre bien la disparition du château et la transformation du domaine.

27. Arch. dép. Seine-et-Marne, Cartes et Plans, 14 FI 4759, plan du château et parc de Chessy, 1740.

28. Nous savons d'après Piganiol, Nouvelle description, op. cit., que la statue avait été dressée dans la cour de l'Hôtel de Ville de Paris et que le roi-même, en 1687, avait ordonné son déplacement. Henri II de Fourcy, qui était alors prévôt des marchands, l'avait amenée à Chessy, en faisant ajouter sur son socle quelques vers de Santeuil. 


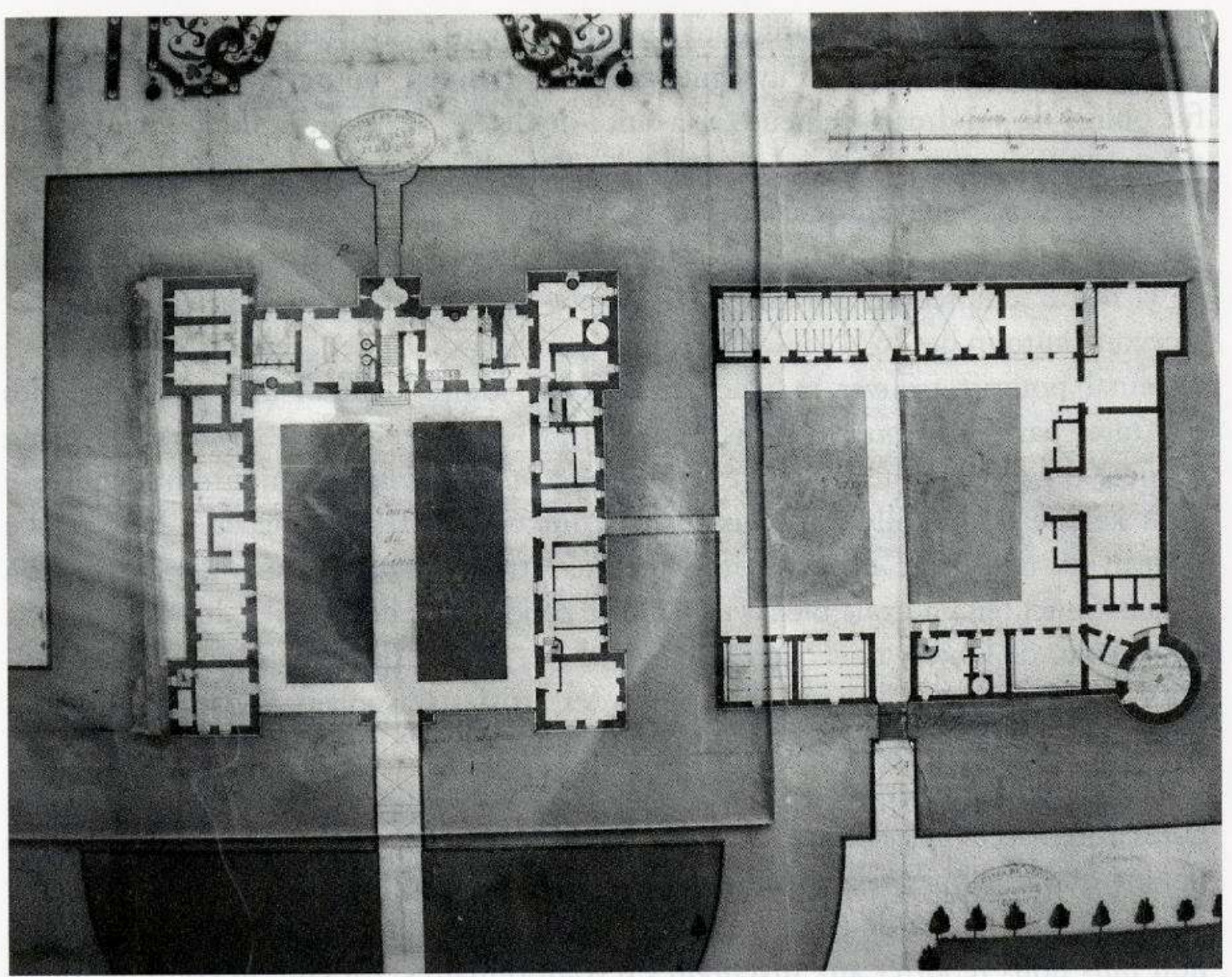

Ill. 5: Anonyme, "Chessy, Plan du Château et des Communs ", Plan du Château et Parc de Chessy, 1740, détail. Arch. dép. de Seine-et-Marne, Cartes et Plans, 14 FI 4759.

niveaux : un étage de service, un étage principal, un étage sous combles, ouvert par des lucarnes et par des œils-de-bœuf. Donc la hauteur du bâtiment restait modeste -3 toises environ, soit moins que 15 mètres - alors que les demeures des financiers des années 1630 se développaient normalement sur deux étages principaux, plus les caves et les combles ${ }^{29}$. Le décor aussi était très simple : des cordons en briques soulignaient le passage d'un niveau à l'autre ainsi que les cadres et les tympans des fenêtres. Les murs enduits contrastent avec le témoignage de Piganiol - qui disait que le château était en brique - et aussi avec les montants en pierre aux coins : il pourrait s'agir d'une intervention du XVIII ${ }^{\mathrm{e}}$ siècle.

Le plan de 1740 nous montre le niveau des communs, avec les cuisines et les caves, ce qui nous empêche d'avoir une idée des appartements : "le château est composé de trois corps de bâtimens, dont deux en ailes sur la cour et un appartemen de jour composé de trois grandes pièces d'un boudoir, et d'une garde robbe, et il y a environ vingt appartemen de maitres dont huit pour de femmes ${ }^{30}$, nous

29. Jean-Marie Pérouse de Montclos, Histoire de l'architecture française, op. cit., p. 215-247.

30. Arch. dép. Seine-et-Marne, 1F177, Mémoires, États de Consistance, pièces diverses concernants la Seigneurie de Chessy, etc., XVIII ${ }^{\mathrm{e}}$ siècle [1767 ?], 6 cc,. n.p. 
dit un État de consistance sans date mais remontant sans doute aux années 16671668, lorsque Maxime de Puységur, fils de la dernière Fourcy, Jeanne Henriette, fut obligé de vendre sa demeure à la suite de dettes ${ }^{31}$. Il faudra donc imaginer les pièces de l'appartement de jour déployées dans le corps-de-logis et dans les deux pavillons, aux deux côtés d'un escalier central - qui devait monter jusqu'aux combles - comme semble nous le montrer la présence de la demi-fenêtre au dessus de la niche sculptée du portail. L'aile gauche abritait peut-être la galerie qui allait recevoir, autour de 1631, le décor peint par Simon Vouet, et dans son pavillon terminal pouvait trouver sa place la chapelle, dont Piganiol attribuait certaines peintures pas moins qu'à Rubens ${ }^{32}$ : faute d'autres documents, Sylvain Kerspern a proposé d'y rattacher un Mariage mystique de Sainte Catherine aujourd'hui conservé dans l'église de Couilly-Pont-aux-Dames, un village très proche de Chessy ${ }^{33}$. Le tableau est une copie de très bonne qualité d'une œuvre rubénienne perdue, déjà à Sans-Souci, et pourrait bien s'agir d'une production de l'atelier du maître ${ }^{34}$. Sa provenance de Chessy doit rester une hypothèse, mais la question se pose de la possibilité que les Fourcy avaient de profiter des artistes travaillant pour la Couronne. Nous allons examiner de façon plus étendue le cas de Simon Vouet.

La documentation sur les décors du château est tout à fait maigre : les inventaires après-décès des Fourcy - surtout celui d'Henri en 1639 - sont décevants, ne notant que quelques tableaux. Alors que Thierry Lhuillier cite des décors à sujet mythologique au rez-de-chaussée, sans nommer ses sources, nous ne pourrons pas non plus nous reporter aux inventaires révolutionnaires. En fait, au contraire de ce qu'on croyait, le château de Chessy n'était pas arrivé à la fin du XVIII ${ }^{\mathrm{e}}$ siècle dans son état d'origine. Un plan dressé en l'An VII (1798-1799) et jusqu'ici ignoré (ill. 6) montre bien sa totale transformation ${ }^{35}$, qu'il faudra dater des années 17821784 lorsqu'un nouveau propriétaire, Joseph Micault de Harvelay entreprend une grande série de travaux dont nous avons retrouvé une partie de la documentation $^{36}$. Alors que le château conservait son plan carré, le corps-de-logis était rebâti à neuf, avec des grandes pièces d'apparat, un double salon central à la façade convexe ; l'étage de service était oblitéré par des escaliers monumentaux, les communs rattachés à la résidence, la chapelle installée à côté du corps-de-logis, selon

31. Thierry Lhuillier, "Chessy ", op. cit., Maxime était très probablement le commanditaire des travaux dont on fait le projet sur les plans de 1740 .

32. Piganiol de la Force, Nouvelle description, op. cit. : "Les peintures de la Chapelle sont encore audessus de celles-la [de la galerie]. Ce sont plusieurs excellents morceaux dont quelques-uns sont de Rubens."

33. Sylvain Kerspern, La Peinture du XVII siècle, op. cit., p. 271-273.

34. Sylvain Kerspern, Tableaux de Couilly-Pont-aux-Dames, Objets d'art... 15 années d'études et de restaurations en Seine-et-Marne (1995-2010). Quelle histoire!, M. Billat éd., Lyon, Lieux Dits Éditions, 2010, p. 120-123.

35. Arch. dép. Seine-et-Marne, $14^{\mathrm{FI}}$ 4968, Plan du Château de Chessy, An 7 (1798-1799), mais les inventaires révolutionnaires remontent au 24 octobre et au 5 décembre 1792.

36. Paris, Arch. nat., T 260, 11-12, Travaux pour la construction des voutes du château de Chessy tant en 1682 et 1683 que pour la distribution de l'aile droite et pour la voute du salon, faite en 1784 . 


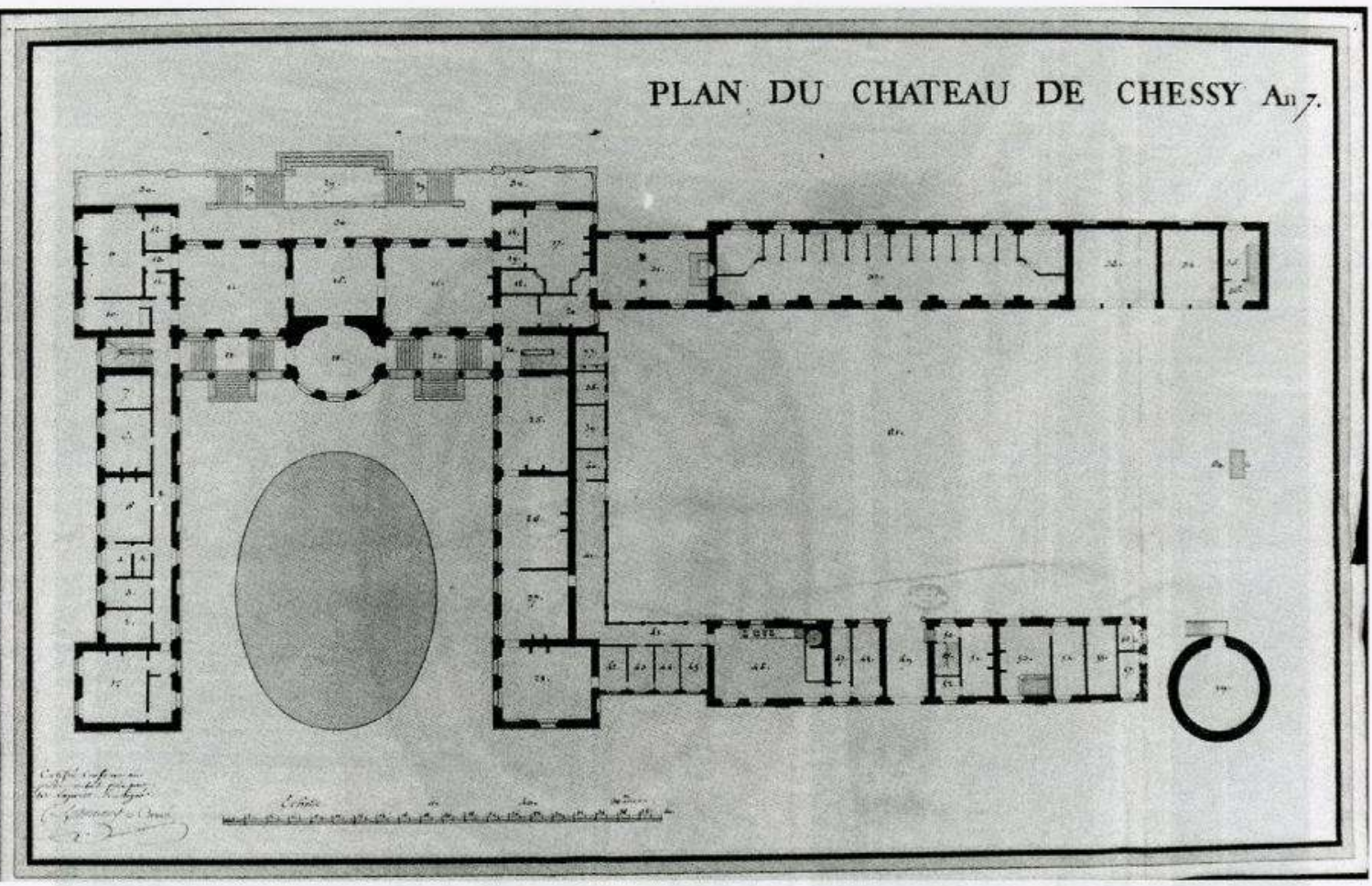

Ill. 6 : Anonyme, "Plan du Château et Parc de Chessy - An 7 », 1798-1799. Arch. dép. de Seine-etMarne, Cartes et Plans, 14 FI 4968.

un plan typique de l'architecture aristocratique au XVIII ${ }^{\mathrm{e}}$ siècle $^{37}$. C'est bien ce nouveau bâtiment qui représente la dernière phase de la vie du château de Chessy et que les inventaires révolutionnaires décrivent. La résidence des Fourcy avait disparu bien avant sa destruction.

\section{Le Surintendant et le peintre: \\ Henri de Fourcy et l'Histoire de Renaud et Armide par Simon Vouet}

Le château de Chessy entièrement perdu, son décor majeur a pourtant survécu. Nous le devons au fils de Jean de Fourcy, Henri († 1638), qui en 1625 lui succédait à la charge de surintendant des Bâtiments. Henri de Fourcy est un personnage majeur dans l'histoire de la peinture française car il fut l'un des premiers commanditaires de Simon Vouet, rentré d'Italie en 1627. Le peintre fut impliqué aussi dans les chantiers du beau-frère du surintendant, le maréchal d'Effiat, et on lui demanda des modèles pour les ateliers des liciers François de la Planche et Charles de Comans ${ }^{38}$.

37. Christophe Morin, Au service du château. L'Architecture des communs en Ille-de-France au XVIII siècle, Paris, Publications de la Sorbonne, 2008, p. 161-212.

38. Denis Lavalle, Vouet, J. Thuiller, B. Brejon de Lavargnée, D. Lavalle dir., cat. expo, Paris, Galeries nationales du Grand Palais, 6 novembre 1990-11 février 1991, Paris, RMN, 1990, p. 512-516. 


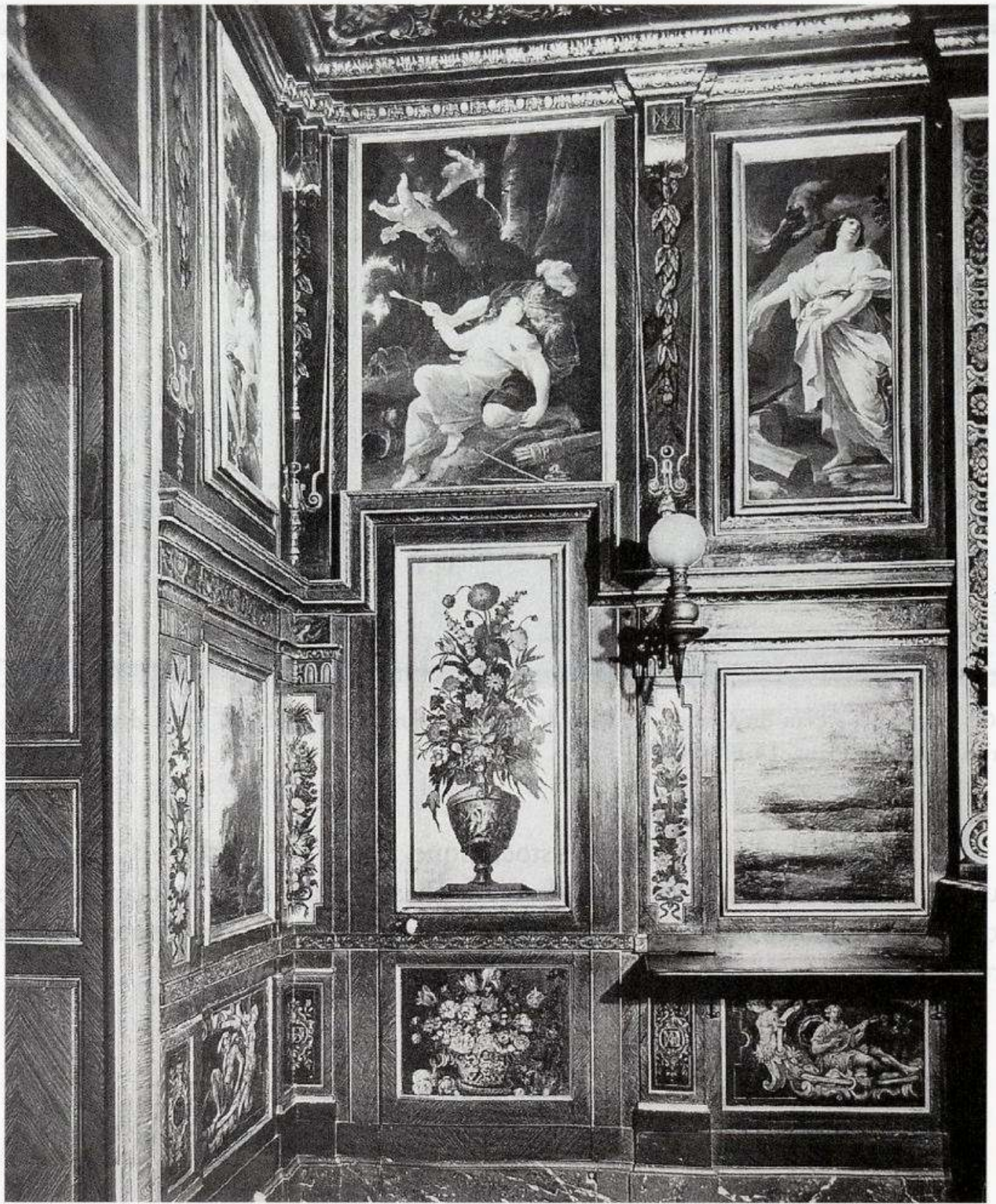

Ill. 7: Simon Vouet, Histoire de Renaud et Armide, huiles sur toile, vers 1630-1631, coll. part. Cl. Guyot de Villeneuve, Louvre.

Pour Henri même, Simon Vouet travailla vers 1630-1631 à Chessy. Dans la galerie du château l'artiste peignit un cycle consacré à l'Histoire de Renaud et Armide: les tableaux, vendus aux enchères en 1767, alors que Maxime de Puységur cherchait à faire face à ses dettes, ont pour cette raison heureusement survécus à la destruction du château ${ }^{39}$. Ils appartiennent aujourd'hui à la collection parisienne Guyot de Villeneuve (ill. 7). Ils ont été remontés dans des boiseries du XVII ${ }^{e}$ siècle

39. Une vente documentée dans les Petites Affiches a été mise en relation avec ces tableaux et la date de 1767 coïncide en effet avec les soucis économiques de Maxime de Puiségur, en précédant d'une seule année la vente du château. Sylvain Kerspern, La Peinture du XVII siècle, op. cit., p. 267. 
qui ne sont pas entièrement cohérentes avec nos peintures. La collection Guyot de Villeneuve étant très difficile à visiter, les tableaux de Simon Vouet sont assez peu connus et très peu étudiés ${ }^{40}$. Ils constituent pourtant l'une des rares séries décoratives réalisées par le peintre en France conservée de façon quasiment intégrale.

Il est difficile de se faire une idée de leur accrochage d'origine. Les tableaux, aux formats et aux dimensions parfois très différents, semblent réfractaires à toute hypothèse de restitution. De riches boiseries et un grand nombre de panneaux mineurs, avec des paysages, des batailles et des arabesques, ornaient sans doute la galerie et ont été dispersés. Plusieurs des panneaux principaux ont été modifiés dans leur format. Ce qui nous reste suffit pourtant à nous donner une idée de l'interprétation vouétienne du récit, qui avait déjà été exploitée par la peinture et la production graphique italiennes que le peintre connaissait très bien, comme nous le prouvent certaines citations iconographiques très évidentes.

Dans chacun des quatorze tableaux, Vouet se penche sur la mise en scène des moments clés de l'histoire : deux tableaux sont consacrés à Armide tombant amoureuse de Renaud et le transportant sur son char; deux tableaux montrent les compagnons de Renaud partis le chercher, alors qu'ils montent dans le bateau de Fortune et qu'il repoussent les avances des nymphes à la Source du Rire; la scène de l'idylle de Renaud et Armide dans le jardin enchanté est suivie par deux tableaux consacrés au repentir du jeune chevalier, qui contemple son reflet dans le bouclier de diamant et abandonne ensuite Armide évanouie au bord de la mer. Finalement, Renaud fait face au fantôme de la magicienne dans la forêt enchantée et, dans le dernier tableau, empêche Armide de se suicider en se réconciliant avec elle.

Armide est en effet la véritable protagoniste du cycle : pas moins de trois tableaux - un quatrième, perdu, est documenté par la tapisserie - la montrent en actrice solitaire, occupée à prononcer des sortilèges ou à commander aux démons. Ainsi, dans la scène où elle trouve Renaud endormi aux bords de l'Oronte, Simon Vouet nous cache son visage - le petit Cupidon à l'arc tendu nous dit le changement de ses sentiments - alors qu'il laisse bien en vue au centre du tableau le poignard levé contre le chevalier. Le peintre s'arrête souvent sur sa beauté et sa sensualité, notamment dans la scène finale de la Réconciliation (ill. 7) où il n'hésite pas à concrétiser de façon flagrante le filigrane érotique des vers du Tasse. Armide est à moitié nue entre les bras de Renaud, vêtu de son armure : leurs têtes sont très

40. Louis Demonts, "Les Amours de Renaud et Armide ", Bulletin de la Société de l'Histoire de l'Art français, 1913, p. 59-78; Rensselaer Lee, Armida's Abandonment. A Study in Tasso Iconography before 1700, De Artibus Opuscula, XL. Essays in honor of Erwin Panofsky, New York 1961, t. I, p. 335-349; Barbara Brejon de Lavergnée, "Simon Vouet, Storie di Rinaldo e Armida ", Torquato Tasso tra letteratura, musica, teatro e arti figurative, A. Buzzoni dir., cat. expo, Ferrare, Nuova Alfa 1985, Bologne 1985, p. 287-289; Barbara Brejon de Lavergnée, Dessins de Simon Vouet, Musée du Louvre. Inventaire général des dessins, Paris, RMN 1987, p. 54-56, n. 21 ; Vouet, op. cit., p. 116-118, ill. 117. Parmi les études les plus récentes qui citent nos tableaux, voir L. Fallay d'Este, "La pittura francese e l'opera del Tasso ", Studi tassiani per il IV centenario della morte di T. Tasso, Sorrente 1995, p. 31-78; Jonathan Unglaub, Poussin and the poetic of painting : pictorial narrative and the legacy of Tasso, Cambridge, Cambridge University Press, 2006, $282 \mathrm{p}$. 
proches, comme s'ils étaient en train de s'embrasser. Le chevalier arrête de sa main droite la flèche que la magicienne avait retournée contre elle-même, sa main gauche s'appuie lourdement sur son sein alors qu'elle fait glisser la sienne sur la jambe de son amant. Pourtant, c'est dans ce même tableau que le sens moralisant du cycle se révèle. Des petits amours dans les airs montrent la couronne nuptiale et la palme de la victoire : la victoire du vrai amour nuptial sur les enchantements sexuels qui avaient enveloppé les deux protagonistes.

Simon Vouet multiplie de pareilles références dans d'autres tableaux. Dans la scène de l'Enlèvement (ill. 8), hormis la magique chaîne de fleurs qui tient Renaud prisonnier de l'enchantement, le public ne pouvait pas manquer de remarquer le grand tournesol tenu par la servante, au centre du tableau, sorte de quatrième personnage à côté des trois qui composent ce groupe en merveilleux chiasme dynamique. Dans le contexte d'un tel épisode de magie, on attend que cette fleur ait une signification négative mais elle est difficile à repérer dans la littérature emblématique de la période, où la capacité qu'a le tournesol de suivre constamment l'astre du jour évoque une fidélité assurée, aussi bien dans le domaine religieux que dans le domaine de l'amour nuptial ${ }^{41}$. Qu'il s'agisse d'un renversement voulu du sens habituel ou de l'insertion d'un indice positif, dans l'une des scènes qui racontent la corruption du héros, le choix de Simon Vouet montre la fonction critique de la mise en image du texte littéraire. Il en va ainsi dans l'ensemble du cycle conçu pour Henri de Fourcy.

À Chessy, l'intention était franchement didactique. Cela se manifeste dans l'importance donnée à Charles et Ubalde résistant à la tentation des nymphes ou par le geste de "bénédiction " que Renaud et ses compagnons adressent à Armide, abandonnée et évanouie, qui modifie de façon substantielle le geste rhétorique qu'avait adopté son modèle, la gravure d'Antonio Tempesta. On a dit que dans ces tableaux Simon Vouet pensait au théâtre - plus que furieuse, la magicienne qui détruit son palais enchanté (ill. 7) semble occupée à chanter un air d'opéra dolent -, mais il est vrai aussi qu'il exprime sa dette envers la rhétorique contemporaine d'origine jésuite, qui n'hésitait pas à adopter la préciosité des gens de la Cour pour conquérir l'attention d'un public aristocratique habitué aux lectures romanesques, bien plus qu'à la rigueur des écrivains classiques et des apologistes chrétiens ${ }^{42}$. Ainsi Simon Vouet s'arrête - avec fascination - sur la fable érotique, mais en véhiculant un message moral très clair qui illustre de façon tout à fait prévisible la victoire de l'ordre chrétien sur les désordres magico-païens.

Cette attention que Henri de Fourcy donnait à l'histoire de Renaud et Armide n'est pas sans rappeler l'importance que les sujets tassiens avaient eue à la Cour de France, pendant les trois décennies précédentes. Marie de Médicis avait proposé son image de courageuse reine des Français et de sage régente sous les masques de Clorinde et de Sofronie, alors que, sous l'influence des Jésuites, la Gerusalemme

41. Sabine Mödersheim, "The Emblem in the context of architecture ", Emblem Scholarship : a tribute to Gabriel Hornstein, P.M. Daly dir., Turnhout, Brepols 2005, p. 171.

42. Marc Fumaroli, L'Âge de l'éloquence, op. cit., p. 247-342. 


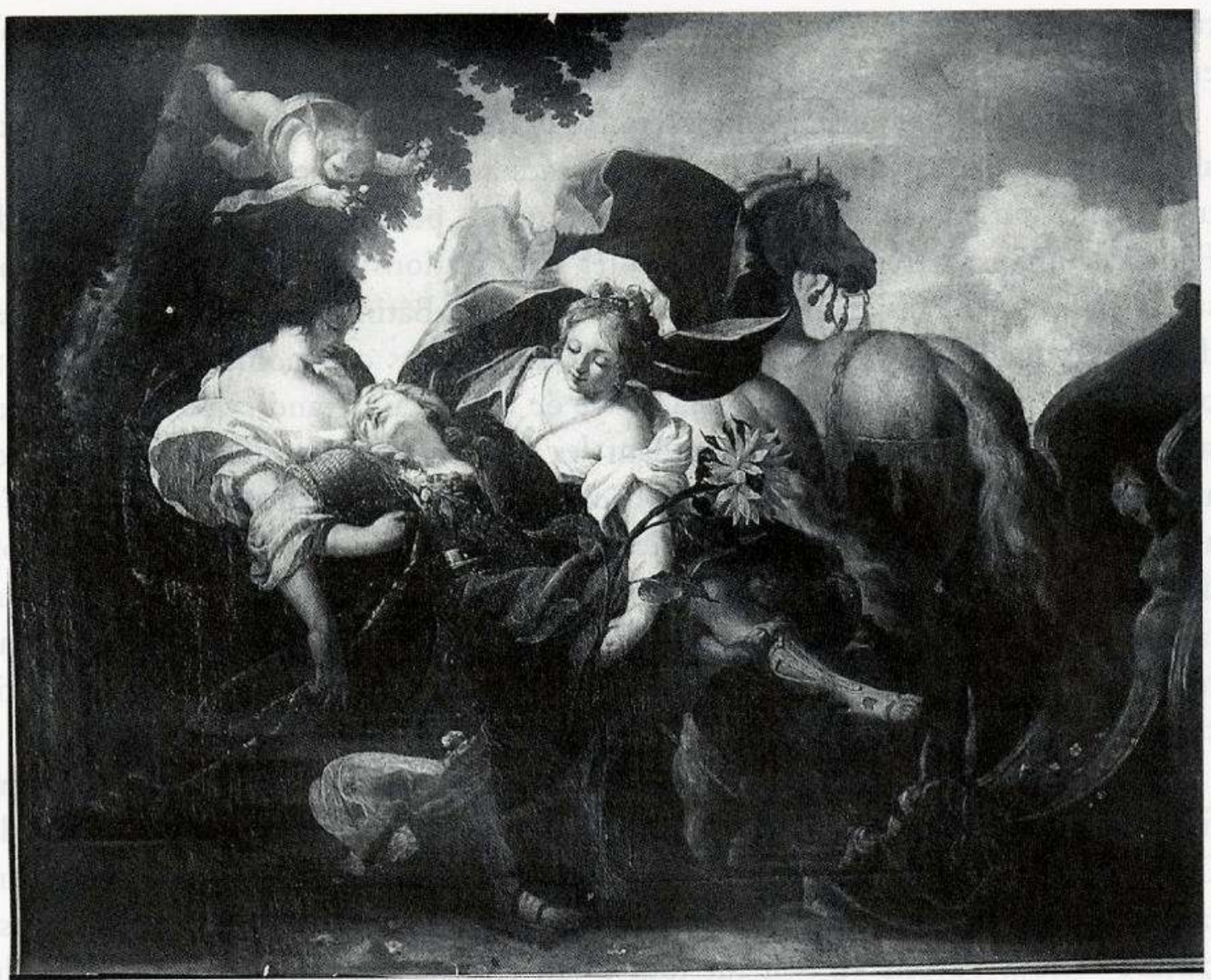

Ill. 8 : Simon Vouet, Armide mène Renaud sur son char, huile sur toile, vers 1630-1631, coll. part. $\mathrm{Cl}$. Guyot de Villeneuve, Louvre.

Liberata devenait une référence pour l'éducation du jeune souverain et que commençait à se lever la voix du père Joseph, qui relançait l'idéal de croisade dont la France et son roi devaient prendre le commandement ${ }^{43}$.

Un tel contexte avait été l'origine du contrechant proposé dans les trois ballets de cour commissionnés par Louis XIII et son favori le duc de Luynes dans les années 1617-1619, où les sujets tirés du Tasse - et de l'Arioste - étaient soumis à une véritable réécriture et interprétation pour marquer l'émancipation du jeune monarque de la tutelle de sa mère. Le premier (1617) montrait un Renaud victorieux des sortilèges d'Armide, dans une mise en scène qui excluait toute réconciliation entre les deux personnages ${ }^{44}$, alors que, dans le troisième (1619), Tancrède

43. Colombe Samoyault-Verlet, "Ambroise Dubois à Fontainebleau ", Le Petit journal des grands expositions, $\mathrm{n}^{\circ}$ 170, 1987 ; Paola Bassani Pacht, "Marie de Médicis et ses artistes ", Le Siècle de Marie de Médicis, F. Graziani, F. Solinas dir., Franco-Italica, n 21-22, 2002, Alessandria, 2002, p. 8194. Pierre Benoist, "Le père Joseph, l'empire Ottoman et la Méditerranée au début du XVII siècle ", Cahiers de la Méditerranée, 71 (2005), http://cdlm.revues.org/index968.html. J'ai donné un essai de lecture de ce phénomène dans ma thèse doctorale.

44. Étienne Durand, Discours au vray du ballet dansé par le Roy, le dimanche XXIXe jour de janvier M.VIc.XVII. avec les desseins, tant des machines \& apparences differentes, que de tous les habits des masques, Paris, Pierre Ballard, 1617, $35 \mathrm{f}$. 
triomphait du fantôme de Clorinde et brisait la magie qui tenait la forêt sous son enchantement ${ }^{45}$.

Le choix de Renaud et Armide en 1630-1631 n'était pas le fruit du hasard, alors que les tableaux de Vouet étaient destinés au château de Chessy, mais aussi à une traduction en tapisserie pour la Couronne et pour les particuliers. Henri de Fourcy connaissait très bien les différentes significations que la Cour donnait aux sujets tassiens : il était le fils d'un surintendant des Bâtiments à qui revenaient les dépenses pour les travaux de décoration et les grandes cérémonies ${ }^{46}$. Ayant remplacé son père, en 1626, il avait déjà essayé de réaliser un grand cycle de tapisseries sur Renaud et Armide, en commandant les modèles à Quentin Varin. Le projet avait échoué avec la mort du peintre (décembre 1626), mais il est significatif qu'il ait été conçu au moment même où Jean Baudoin publiait sa nouvelle traduction de la Gerusalemme. Henri de Fourcy finalement avait réussi quelques années plus tard à réaliser ce cycle qu'il confia à Simon Vouet. Il était destiné à une fortune remarquable: tissé trois fois pour la Couronne et plusieurs fois pour des particuliers, il arriva très tôt à Rome, chez les Barberini ${ }^{47}$.

Henri de Fourcy était, comme son père, un représentant de la robe d'origine financière, étranger au monde de la noblesse d'épée, quoiqu'étroitement lié à la Cour par ses charges et par tradition familiale. Il occupait aussi une position importante dans le monde parlementaire, bien que, par son histoire personnelle et sa culture - nous l'avons vu - il fût tout à fait éloigné des milieux robins issus du barreau et de l'université, liés à la République des Lettres et marqués par des tendances gallicanes. Il avait obtenu la charge de président de la Chambre des comptes le 16 septembre $1631^{48}$, au lendemain de la fuite de Marie de Médicis, en plein renouvellement des charges entrepris par Richelieu afin d'éliminer les soutiens de la reine mère. Le beau-frère d'Henri, d'Effiat, l'un des fidèles du Cardinal, ne fut peut-être pas étranger à cette nomination, qui témoigne de la confiance que le premier ministre faisait à Fourcy, ainsi que de la position du dernier depuis le début de la crise de 1628. Mais cette situation ne semble pas avoir empêché Fourcy de montrer un point de vue autonome dans le théâtre politique du début des années 1630.

Il agissait avec les outils qui étaient les siens, c'est-à-dire la création d'œuvres d'art pour la Couronne. Il savait bien qu'à travers la déclinaison d'un sujet connu de tous, les images exerçaient leur influence sur le public : le monarque, bien sûr, mais aussi les aristocrates, grands et petits, à la recherche de décors somptueux. Il agissait en employant un artiste maîtrisant parfaitement la muta eloquentia de la

45. Giovanni Careri, Geste d'amour et de guerre. La Jérusalem délivrée, images et affects (XVI XVIII ${ }^{e}$ siècle), Paris, Éd. EHESS, 2005, p. 204-214.

46. Bernard Barbiche, "Henri IV et la surintendance des Bâtiments", op. cit., p. 20-21.

47. Vouet, op. cit., p. 512-516. La "Série Barberini" est conservée aujourd'hui au Flint Museum of Art (Ohio).

48. Bernard Barbiche, "Henri IV et la surintendance des Bâtiments ", op. cit., p. 20-21. 
peinture. Il agissait, enfin, en proposant un sujet tout à fait connu à la Cour, mais ramené à une sorte d'orthodoxie tassienne - après combien de variations au cours des décennies précédentes - et interprété selon une lecture qui soulignait la pacification dynastique.

Cette portée idéologique découle de la mise en scène et des détails que Simon Vouet y intégra. Au lendemain de la crise qui aboutit à la Journée des Dupes, une nouvelle phase de la monarchie française s'ouvrait avec le départ - habilement forcé par Richelieu - de la reine mère, un départ qui deviendra un exil ${ }^{49}$. La Cour en avait été frappée, alors même qu'Anne d'Autriche se trouvée compromise dans cette dernière " cabale " qui avait entraîné la crise définitive dans la maison royale. Dans ce contexte, la démarche d'Henri de Fourcy ne pouvait pas revenir au hasard, le sujet et ses implications idéologiques étant trop connus.

Une telle opération avait évidemment aussi une valeur personnelle, qui nous échappe, car elle était liée à l'endroit où le cycle était installé et adressée à l'entourage personnel du surintendant, un petit groupe restreint de familiers et de personnages du même milieu de noblesse liée à l'administration. Nous en trouvons trace parmi les noms qui signent les pièces liminaires des ouvrages du prélat Charles de Titreville, protonotaire et professeur d'hébreu au Collège royal. Ses livres, publiés entre 1637 et 1646, sont accompagnés de poèmes écrits par les fils de Henri de Fourcy - Henri II, Jean III, Claude - par François de Grenaille, par Guillaume Colletet. Les mêmes noms qui apparaissent parmi les soutiens du philosophe et philologue Émeric Crucé - auteur du Nouveau Cynée et traducteur de Stace lorsqu'il se trouvera au milieu d'une polémique littéraire avec Gaspard Gevaerts - lié au salon de Henri de Mesmes - puis avec Johann Friederich Gronovius, lié aux frères Dupuy ${ }^{50}$ : qui est finalement, encore une fois, aussi une polémique entre deux milieux de magistrats aux origines différentes, les financiers d'une part, les savants robins de l'autre ${ }^{51}$. Charles de Titreville était ami intime d'Henri dont il écrivit l'éloge funèbre en 1638. Il avait aussi écrit un poème latin, Chessiacum. Ad D. Henricum de Fourcy ${ }^{52}$, qui tissait la louange du château de Chessy, peut-être la meilleure clé de lecture pour comprendre le château et son décor. Mais le Chessiacum est perdu, nulle trace ne nous en reste.

Reste le cycle peint par Vouet. Bien que sorti de son contexte d'origine et sans doute incomplet, il montre Henri de Fourcy occupé à réaliser un décor qui ne pouvait pas passer inaperçu. Car revenir sur l'histoire de Renaud et Armide en la

49. Jean-Claude Dubost, Marie de Médicis. La reine dévoilée, Paris, Payot, 2009, p. 784-804.

50. Émeric Crucé, Il nuovo Cinea. Per la pace universale, A.M. Lazzarino del Grosso dir., Naples, Guida 1979, p. 34 et p. 50 sq.

51. J'ai proposé une étude sur l'entourage culturel des Fourcy dans mon essai Tra la Corte e l'Accademia. Il micro-contesto di alcuni entourages nobiliari all'epoca di Luigi XIII : il caso dei de Fourcy, Le Virtuose adunanze. La cultura accademica tra XVI e XVII secolo, actes du colloque international, Latina 16-17 février 2012, Clizia Gurreri dir., à paraître.

52. Thierry Lhuillier, Chessy, op. cit. ; Sylvain Kerspern, La Peinture du XVII e siecle, op. cit., p. 266. 
lisant de façon positive au tournant de 1630-1631, c'était adresser un message sans équivoque et qui n'était pas forcément en phase avec l'atmosphère de reddition de comptes voulue par Richelieu, désormais arbitre incontesté de la politique française. Un message de rédemption et de réconciliation que tout le monde pouvait comprendre, le cardinal le premier. Qu'il le partageât, cela n'est pas du tout certain.

Gabriele QUARANTA

docteur en histoire de l'art

Sapienza Università di Roma

université Paris 1-Panthéon-Sorbonne

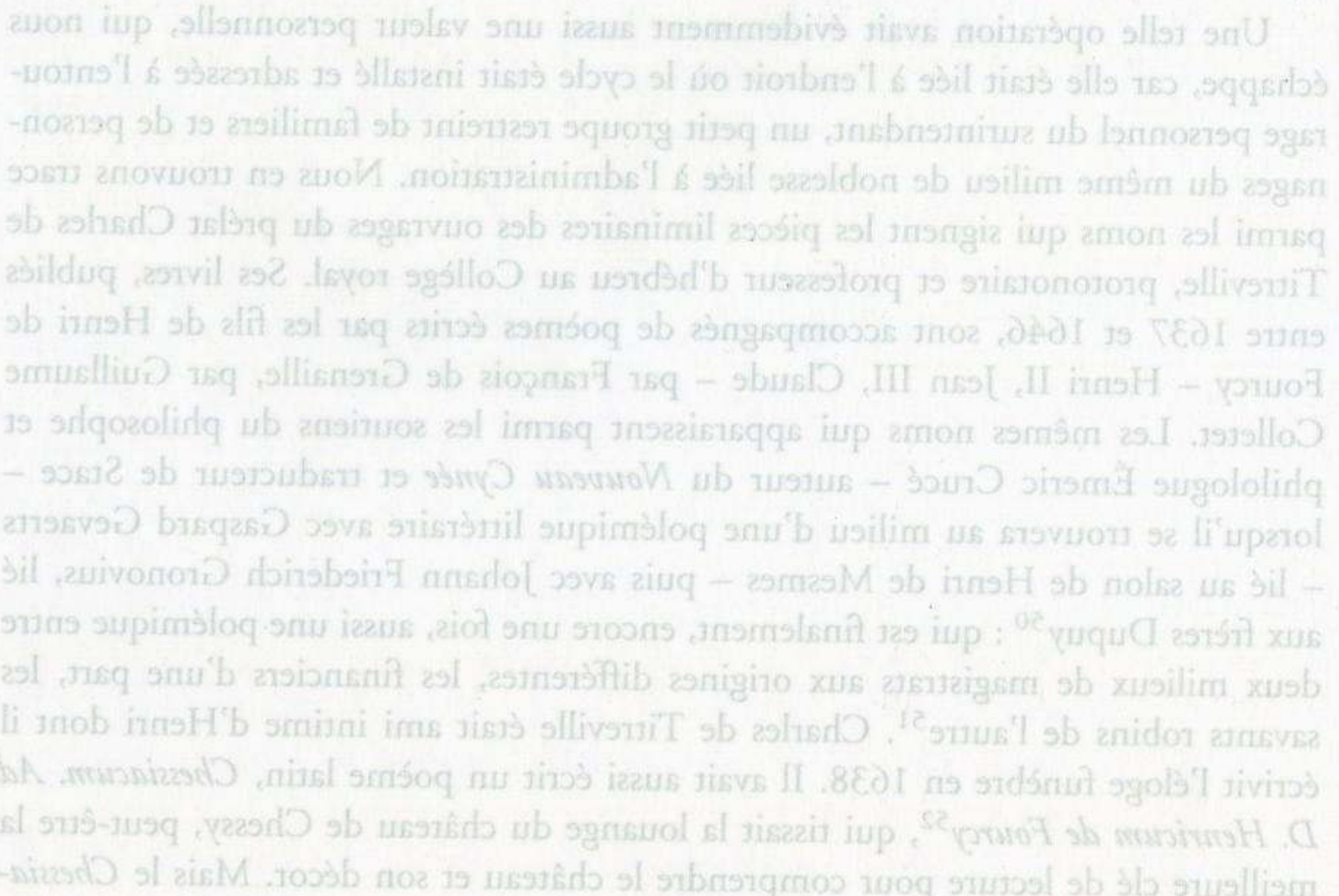

